\title{
Evaluation De La Prise En Charge De La Malnutrition Aigüe Sévère Dans Le Service De CRENI Du Centre Hospitalier Régional (CHR) De Niamey
}

\author{
D. Bonkano Baoua,
}

Service de pédiatrie, centre hospitalier régional poudrière de Niamey

\author{
M. Garba, \\ Ms Maman Moussa, \\ N. Naneito Moussa,
}

Service de pédiatrie, Hôpital National de Lamordé de Niamey

\section{Kamaye,}

Département de Médecine et Specialités médicales, Université Abdou

Moumouni de Niamey

Service de pédiatrie, Hôpital National de Lamordé de Niamey

Doi:10.19044/esj.2020.v16n24p233 URL:http://dx.doi.org/10.19044/esj.2020.v16n24p233

\section{Résumé}

Introduction L'objectif de notre étude est d'évaluer la prise en charge de la malnutrition aigüe sévère dans le centre de récupération nutritionnelle intensive du centre hospitalier régional de Niamey.

Matériels et méthodes : nous avons mené une étude prospective de soins courants réalisée entre le 1er janvier 2016 et le 31 décembre 2016 en incluant les enfants de 0 à 59 mois qui présentaient un rapport poids/taille $\leq$ $3 \mathrm{Z}$ score et ou des œdèmes de dénutrition chez lesquels une malformation cardiaque ou néoplasies ou autres maladies chroniques étaient éliminées. Le protocole national de la récupération nutritionnelle est divisé en phase 1 pendant laquelle les patients sont mis sous le lait F-75 $(130 \mathrm{ml} / \mathrm{kg} / \mathrm{j})$, en phase de transition avec la mise sous le F-100 $(130 \mathrm{ml} / \mathrm{kg} / \mathrm{j})$ et en phase 2 pendant laquelle les patients sont mis sous l'alimentation thérapeutique près à l'emploi et ou le F-100. Le passage d'une phase à une autre est bien codifié par le protocole.

Résultats : Durant la période de l'étude, 5773 patients ont été recensés, parmi lesquels 814 étaient atteints de MAS soit une fréquence de $14,1 \%$ de toutes les pathologies rencontrées dans notre service. Le sexe masculin était prédominant $(52,5 \%)$ avec l'âge moyen des patients à $14,4 \pm 9,14$ mois. Les patients issus de familles à niveau de vie faible 
représentaient $75 \%$. Le marasme représentait $86,30 \%$ des cas de malnutrition, le kwashiorkor $5,03 \%$ et la forme mixte $8,70 \%$. La grande majorité des malades $(91,52 \%)$ avait un $\mathrm{Z}$ score $\leq-3$. L'ECBU était positive chez 85 patients. L'E Coli représentait $87 \%$ des germes rencontrés. Les facteurs de comorbidité étaient le paludisme $(88,8 \%)$; les pneumopathies $(5,28 \%)$ et le VIH (1,4\%). Les principales complications rencontrées au cours de l'hospitalisation étaient la déshydratation (26,5\%), la détresse respiratoire (6,14\%) et l'hypothermie (3,2\%). La durée moyenne de séjour hospitalier était de $15,7 \pm 5,2$ jours et $12 \%$ des patients avaient un séjour de plus de 20 jours. Le gain pondéral moyen journalier était de $12,5 \pm 3,9 \mathrm{~g} / \mathrm{kg} / \mathrm{jour}$. Nous avions enregistré 66 cas de décès $(8,1 \%)$.

Conclusion: L'étude montre une prévalence très élevée du marasme, une prédominance des enfants de moins de 24 mois et une mortalité élevée chez les nourrissons. Promouvoir une bonne diversification alimentaire chez le nourrisson permettrait d'améliorer la situation.

Mots clés : Malnutrition Aigüe Sévère, Évaluation, Prise En Charge, Niamey, Niger

\title{
Evaluation Of The Management Of Severe Acute Malnutrition In The CRENI Department Of The Regional Hospital (CHR) Of Niamey
}

\author{
D. Bonkano Baoua,
}

Service de pédiatrie, centre hospitalier régional poudrière de Niamey

$$
\text { M. Garba, }
$$

Ms Maman Moussa,

N. Naneito Moussa,

Service de pédiatrie, Hôpital National de Lamordé de Niamey

M. Kamaye,

Département de Médecine et Specialités médicales, Université Abdou

Moumouni de Niamey

Service de pédiatrie, Hôpital National de Lamordé de Niamey

\section{Abstract}

Background: The objective of our study is to evaluate the management of severe acute malnutrition in the intensive nutrition recovery center of the Regional Hospital Center of Niamey. 
Methods: we carried out a prospective study of routine care performed between January 1, 2016 and December 31, 2016 including children from 0 to 59 months who presented a weight / height ratio $\leq-3 \mathrm{Z}$ score and or edema of undernutrition in which a heart malformation or neoplasms or other chronic diseases were eliminated. The national protocol for nutritional recovery is divided into phase 1 during which patients are put on F-75 milk (130ml / kg / d), in the transition phase with putting on F-100 (130ml / kg / d ) and in phase 2 during which the patients are put on therapeutic food ready for use and / or F-100. The transition from one phase to another is well codified by the protocol.

Results: During the study period, 5,773 patients were identified, among which 814 had SAM, ie a frequency of $14.1 \%$ of all pathologies encountered in our department. Male sex was predominant (52.5\%) with the mean age of patients at $14.4 \pm 9.14$ months. Patients from families with a low standard of living accounted for $75 \%$. Marasmus accounted for $86.30 \%$ of malnutrition cases, kwashiorkor $5.03 \%$ and mixed form $8.70 \%$. The vast majority of patients $(91.52 \%)$ had a $\mathrm{Z}$ score $\leq-3$. The urine culture was positive in 85 patients. E Coli accounted for $87 \%$ of the germs encountered. The comorbid factors were malaria (88.8\%); pneumonia (5.28\%) and HIV (1.4\%). The main complications encountered during hospitalization were dehydration (26.5\%), respiratory distress (6.14\%) and hypothermia (3.2\%). The mean length of hospital stay was $15.7 \pm 5.2$ days and $12 \%$ of patients had a stay of more than 20 days. The mean daily weight gain was $12.5 \pm 3.9 \mathrm{~g} / \mathrm{kg}$ / day. We had recorded 66 cases of death (8.1\%).

Keywords : Severe Acute Malnutrition, Assessment, Management, Niamey, Niger

\section{Introduction}

La malnutrition aigüe sévère (MAS) représente encore un problème de santé publique dans les pays en développement. Dans le monde plus de 20 millions d'enfants sont exposés à la malnutrition aigüe et chronique et environ 1 million de décès d'enfants dans le monde sont imputables à la malnutrition (United Nations of International Children's Emergency Fund [UNICEF] et Organisation Mondiale de la Santé [OMS], 2012). Selon une étude menée par Burza, Mahajan, Marino et al. (2015), comme dans le rapport (UNICEF et OMS 2009), les enfants atteints de MAS ont plus de risque de tomber malade et contribuent à l'augmentation de la morbidité et de la mortalité infantile. Dans le monde, plus de $90 \%$ des cas de malnutrition sont recensés dans 36 pays dont le Niger (UNICEF, 2015). Au Niger la malnutrition demeure un problème de santé publique car le pays fait face aux déficits alimentaires chroniques observés dans plusieurs zones vulnérables. Elle résulte également 
des maladies infectieuses et parasitaires (paludisme, rougeole, diarrhées) fréquentes chez l'enfant, dans un contexte de système de soins déficients, de connaissance insuffisante des besoins nutritionnels du jeune enfant et de pratiques culturelles inadaptées aux besoins du jeune enfant. Selon l'enquête Démographique et de Santé et à Indicateurs Multiples 2012, la MAS concerne $18 \%$ des enfants dont 6\% sous la forme sévère (Institut National de la Statistique [INS], 2012). Pour lutter efficacement contre la MAS depuis 2005, le Niger a mis en place des centres de récupération nutritionnelle ambulatoire pour malnutris modérés (CRENAM) et des centres de récupération nutritionnelle intensive (CRENI) pour les malnutris sévères sur l'ensemble du territoire. En plus le pays a adopté un protocole national de prise en charge de la malnutrition à l'instar de tous les pays concernés par le problème. Ce protocole a été révisé en 2012 pour le standardiser aux normes internationales. L’objectif principal de notre étude est d'évaluer l'efficacité de la prise en charge de la malnutrition aigüe sévère au CRENI du centre hospitalier régional (CHR) de Niamey, Niger.

\section{Matériels et méthodes}

Il s’agissait d’une étude prospective de soins courants réalisée sur une période d'un an allant du $1^{\mathrm{er}}$ janvier 2016 au 31 décembre 2016. Elle était menée dans le CRENI du CHR de Niamey. Le CHR est un hôpital de niveau secondaire qui dessert la ville de Niamey et ses environs. L’unité de récupération nutritionnelle a une capacité de 26 lits d’hospitalisation courante et 3 lits de soins intensifs. L'étude avait inclus tous les enfants de 0 à 59 mois hospitalisés pour MAS dont le test de l'appétit est médiocre ayant un rapport Poids/Taille $(\mathrm{P} / \mathrm{T}) \leq-3 \mathrm{Z}$ score ou bien présentant des œdèmes de dénutrition bilatéraux et symétriques. Nous n'avions pas inclus dans l'étude les enfants atteints de malformation cardiaque, de cancer ou d'autres maladies chroniques. Nous avions défini le marasme comme une émaciation ou dénutrition sévère avec disparition totale du tissu graisseux et ne présentant pas des œdèmes dont le rapport $\mathrm{P} / \mathrm{T} \leq-3 \mathrm{Z}$ score. Le kwashiorkor est défini comme une dénutrition présentant des œdèmes bilatéraux et dont le pannicule adipeux subsiste. La forme mixte est l'association du marasme et du kwashiorkor. Le cas spécial est un enfant malnutri aigu sévère qui est âgé de moins de 6 mois avec un poids inférieur à 3kg et/ou un rapport $\mathrm{P} / \mathrm{T}<-3 \mathrm{Z}$ score. La récupération nutritionnelle selon le protocole en vigueur est divisée en trois phases. La première phase pour stabiliser les patients, elle dure en moyenne une semaine. Au cours de cette phase les patients sont mis sous le lait F-75 $(130 \mathrm{ml}=>100 \mathrm{kcal})$ en raison de $130 \mathrm{ml} / \mathrm{kg} /$ jour. Ce produit permet d'amorcer le rétablissement des fonctions métaboliques et l'équilibre nutritionnel. La phase de transition qui prépare le patient au traitement de phase 2 ou au traitement en ambulatoire pour malnutris sévères (CRENAS). Au cours de 
cette phase le F-100 (100 ml =>100 kcal) est donné en raison de 130 $\mathrm{ml} / \mathrm{kg} /$ jour. La dernière phase est la phase 2 où le patient est mis sous alimentation thérapeutique près à l'emploi (ATPE) et ou le F-100. Le passage d'une phase à une autre est bien codifié par le protocole. Pour le besoin de l'étude nous avons défini le niveau de vie faible la situation d'un ménage dont les deux conjoints n’ont pas d'activité professionnelle stable régénératrice de revenus. Le niveau de vie moyen est défini comme la situation d’un ménage dont au moins un conjoint a une activité professionnelle stable régénératrice de revenus. Le niveau de vie élevé est la situation d'un ménage dont au moins un conjoint exerce une activité professionnelle au niveau tertiaire, ou est un cadre de l'administration ou un grand commerçant. Un patient est déclaré guéri quand il atteint un rapport $\mathrm{P} / \mathrm{T} \geq-1,5$ et ou absence des œdèmes nutritionnels pendant 14 jours. L'abandon est une absence du patient sur son lit d'hospitalisation pendant plus de 48 heures. Le transfert est une référence d'un patient vers un hôpital de niveau supérieur pour des raisons médicales. L’échec de récupération nutritionnelle est une stagnation ou une chute pondérale persistant pendant au moins une semaine.

Pour chaque patient nous avons recueilli les caractéristiques sociodémographiques (âge, sexe, provenance, profession des parents), caractéristiques anthropométriques (poids à l'admission, taille, rapports poids/taille), antécédents médicaux (type d'alimentation, âge de diversification alimentaire, antécédent d'hospitalisation, vaccination), type malnutrition à l'admission, les complications médicales à l'admission et au cours de l'hospitalisation, le bilan biologique et radiologique, le suivi de l'appétit et des œdèmes, le poids quotidien et le mode de sortie. Les données collectées ont été saisies et analysées à l’aide des logiciels Epi-Info 7.1 du CDC d'Atlanta et STATA.12. Nous avions utilisé les mesures de tendances centrales et de dispersion, et les proportions pour la phase descriptive. Le test de $\mathrm{Chi}^{2}$ a été utilisé pour comparer les variables qualitatives. La différence a été jugée statistiquement significative au seuil de 5\%. L’Odds ratio et l'intervalle de confiance à 95\% ont été calculés pour la quantification du risque, et estimer la liaison entre la malnutrition et plusieurs paramètres.

\section{Résultats}

Durant la période de l'étude 5773 patients ont été recensés, parmi lesquels 814 étaient atteints de MAS soit une fréquence de 14,1\% de toutes les pathologies rencontrées dans notre service. Parmi les 814 atteints de MAS, il y’a 87 cas spéciaux soit une proportion de 10,7\%. L’âge moyen des patients

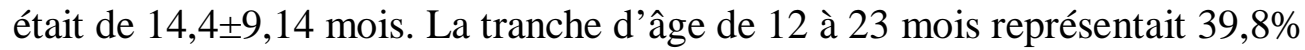
des cas. Le sexe masculin représentait 52,5\% de notre échantillon. Trois quart des patients $(74,2 \%)$ provenaient de la ville de Niamey. Dans la grande majorité des cas $(91,30 \%)$ les mères des patients malnutris étaient non 
scolarisées. Soixante-quinze pour cent des patients étaient issus de famille à niveau de vie faible et $25 \%$ de famille à niveau de vie moyen. L'ensemble des caractéristiques sociodémographiques des patients sont résumées dans le tableau 1. Plus de la moitié $(53,31 \%)$ des patients étaient référés des dispensaires périphériques, 43,12\% des patients étaient admis directement dans l'unité au cours des consultations de routine et 3,57\% étaient des réadmissions. Le pic d'admission a été observé au cours du mois de juillet et le mois de février a connu le plus faible taux d'admission (figure 1). Seulement 29,5\% des patients avaient bénéficié d'un allaitement maternel exclusif jusqu'à 6 mois, 11,1\% avaient eu une bonne diversification alimentaire. Sur les 814 patients, 56,1\% avaient une vaccination complète selon le programme national des immunisations et $67,2 \%$ étaient vaccinés contre la rougeole. Environ 19\% des patients avaient un antécédent d'hospitalisation médicale. Cent douze malnutris sur 814 (13,75\%) avaient des œdèmes de dénutrition à l'admission. Le marasme représentait le type de malnutrition le plus enregistré (86,30\%) des cas suivi de la forme mixte (8,70\%) et du Kwashiorkor (5,03\%). Le poids moyen des patients à l'admission était de 5847, $2 \pm 580,8$ grammes et $56,6 \%$ avaient un poids $\leq 6000$ grammes. La taille moyenne des patients à l'admission était de 69,4 centimètres avec des extrêmes allant de 41,5 à 101 centimètres. Le périmètre brachial moyen à l'admission était de $10,5 \pm 0,51 \mathrm{~cm}$. La grande majorité des malades $(91,52 \%)$ avait un $\mathrm{Z}$ score $\leq-3$. Le tableau 2 résume les valeurs du $\mathrm{Z}$ score des patients à l'admission et à la sortie. $\mathrm{A}$ l'hémogramme 15,1\% des patients avaient un taux d'hémoglobine $<7 \mathrm{~g} / \mathrm{dl}$. Parmi lesquels $50 \%$ avaient une anémie sévère (taux d'hémoglobine $<5 \mathrm{~g} / \mathrm{dl}$ ). L'étude cytobactériologique des urines (ECBU) a été demandée chez 205 enfants qui présentaient des signes infectieux. Elle était positive chez 85 patients. L'E. coli était retrouvé chez $87 \%$ de culture positive suivi du Streptococcus SPP (7\%). L'examen parasitologique des selles effectuée chez 394 patients avait objecté 8 cas de Trichomonas intestinalis, 8 cas Entamoeba histolitica et 7 cas de Giardia lamblia. La goutte épaisse était demandée de manière systématique chez tous les patients. Elle était positive chez $88,8 \%$ des patients. La sérologie VIH était positive chez 11 enfants (1,4\%). La radiographie pulmonaire avait montré des images de pneumopathie chez $5,28 \%$ malades. Parmi lesquels deux patients étaient atteints de la tuberculose confirmée par la recherche de bacille amino-alcoolo-résistant (BAAR) dans le liquide de tubage gastrique. Les principales complications (tableau 3) étaient la déshydratation (26,5\%), la détresse respiratoire $(6,14 \%)$ et l'hypothermie (3,2\%). La durée moyenne de séjour hospitalier était de 15,7 $\pm 5,2$ jours. Douze pourcents avaient une durée d'hospitalisation de plus de 20 jours. Le gain pondéral moyen journalier était de $12,5 \pm 3,9 \mathrm{~g} / \mathrm{kg} / \mathrm{jour}$. Cent quatre-vingts trois patients $(22,48 \%)$ avaient un gain pondéral supérieur à $15 \mathrm{~g} / \mathrm{kg} / \mathrm{jour}$. 
Le taux d’abandon était de 1,7\%. Trois cent-soixante-deux patients (45\%) étaient transférés au CRENAS pour compléter la récupération nutritionnelle. Nous avions enregistré 66 cas de décès (8,1\%). Environ 64\% des décès survenaient dans les premières 72 heures. Le tableau 4 résume les causes probables de décès des patients. Les enfants âgés de moins de 24 mois représentaient $81,8 \%$ des décès et les enfants atteints de marasme représentaient $87,9 \%$ des décès. L’analyse uni-variée avait montré que le marasme était plus fréquent en zone rurale $(\mathrm{OR}=0,58$; IC à $95 \%$ : 0,4 et 0,9 et $\mathrm{P}=0,007)$. Le sexe masculin était plus exposé aux complications $(\mathrm{OR}=0,8$; IC à $95 \%$ : 0,56 et 1,$04 ; \mathrm{P}=0,04)$. Les garçons avaient un séjour hospitalier plus long que les filles $(\mathrm{P}=0,001)$. Mais ils ont un taux de récupération nutritionnelle plus élevé que les filles. Les enfants malnutris incomplètement vaccinés avaient plus de risque de décéder au cours de l'hospitalisation que les autres avec OR à 1,59 [IC 0,9; 2,5 et $\mathrm{p}=0,001$ ].

\section{Discussion}

En plus de son caractère monocentrique et la taille relativement petite de notre échantillon ainsi que quelques difficultés comme le problème d'archivage des données et la pratique de la pesée des patients par des intervenants différents qui constituent les principales limites de l'étude. Elle avait permis de montrer que la MAS a une incidence de 14,1\% dans notre service, que la malnutrition survient à un âge précoce et que la majorité des enfants malnutris étaient issus de parents non scolarisés. Les garçons étaient les plus affectés par la malnutrition (52,5\%) dans notre échantillon. MweneBatu P et al (2020) et Dembélé I et al. (2019) rapportent aussi cette prédominance masculine d'envions 57\% dans les 2 études. Dans une série rapportée par Black et al. (2008) il y’a une prédominance de la MAS chez les enfants de sexe féminin. Par contre Madzorera I et al (2018) et Zoungrana B et al (2019) rapportent une égalité des sexes dans la survenue de la MAS. La disparité des résultats pourrait être liée à la variété des pratiques culturelles dans l'alimentation des enfants en Afrique. L'âge moyen de nos patients était de 14,4 $\pm 9,14$ mois. Des résultats similaires sont rapportés par Zoungrana B au Burkina Fasso (2019) et F. Nguefack au Cameroun(2015) avec respectivement 12 et 13 mois. Par contre dans la série de Mwene-Batu P et al (2020) l’âge médian était de 41 mois. Comme dans la série rapportée par Saaka, Osman, Amponsem et al au Ghana (2015), nous avons recensé un pic d'admission durant les mois de juin et juillet. Cette période appelée communément période de soudure, coïncide avec le pic d'infestation paludéenne et de pénurie alimentaire dans les zones rurales au Niger. La grande majorité de nos patients (82,5\%) avait un âge inférieur à 24 mois. des résultats similaires ont été rapportés par Zoungrana B et al (2019), Madzorera I et al (2018), Nimpagaritse et al (2019). Cela démontrait la survenue précoce 
de la malnutrition dans nos contrées. Le plus souvent liée à une mauvaise pratique de la diversification alimentaire chez le nourrisson comme en témoigne les 11,1\% seulement d'une bonne diversification alimentaire dans notre étude ou un déficit d’apport d'aliments riches chez les nourrissons. Ce même constat a été rapporté par Chaand I et al (2019) en Inde. Le marasme était la forme prédominante de malnutrition dans notre échantillon (86,30\%) comme dans l'étude de Dembélé I et al (2019) au Mali avec 94,15 \%. En revanche le kwashiorkor était la forme prédominante de la malnutrition dans la série de Mwene-Batu P et al (2020) et Mbusa Kambale R et al (2016) en république démocratique du Congo. La fréquence élevée du marasme dans notre étude peut être liée à une carence alimentaire globale chez les enfants dès leur jeune âge. Du reste $75 \%$ de nos patients étaient issus de famille à revenu faible. La pauvreté limite fortement la capacité des parents à pourvoir efficacement aux besoins nutritionnels des enfants. Dans une étude rapportée par Madzorera I et al (2018) la pauvreté et l'illettrisme des parents sont cités comme des facteurs de risque très importants dans la survenue de MAS. Les principaux facteurs de comorbidité dans notre étude étaient le paludisme, l'infection urinaire, l'anémie, les parasitoses intestinales et la pneumonie. Ces mêmes constats ont étés rapportés par Kanan et al (2016). Ces maladies, souvent à l'origine de diarrhée chez l'enfant, et sont citées comme facteurs précipitants de la survenue de MAS par Singh et al (2014). Environ 30\% de nos patients avaient bénéficié d'un allaitement maternel exclusif pendant les six premiers mois. Dans une étude rapportée Kabore A et al (2019) réalisée chez les enfants de moins de six mois, seulement 17,46 \% étaient nourris exclusivement au sein. L'allaitement maternel permet un bon développement cognitif et sensoriel de l'enfant et le protège contre les maladies infectieuses et chroniques. Il réduit aussi la mortalité due aux maladies de l'enfance comme la diarrhée et la pneumonie. La durée moyenne d'hospitalisation de nos patients était de 15,7 jours. Des durées similaires ont été rapportées par Munthali et al (2015) et Singh et al (2014). Un suivi plus d'un 30 jours a été rapporté par Mengesha MM et al (2016) dans une revue éthiopienne par contre Zoungrana $\mathrm{B}$ et al retrouve une durée moyenne d'hospitalisation plus courte de 09 jours. La forte demande d'hospitalisation contribue souvent à raccourcir la durée d'hospitalisation. Ce qui a permis d'avoir un taux de guérison de $70 \%$. Le gain pondéral journalier de nos patients était de 12,5 $\pm 3,9 \mathrm{~g} / \mathrm{kg} / \mathrm{jour}$. Singh et al (2014) ont rapportés des résultats similaires dans la littérature. Zoungrana B et al (2019) avancent un gain pondéral journalier meilleur de 29 g/kg/jour. Par contre dans la série de Mbusa Kambal R et al(2016) le gain pondéral était modeste à $7 \mathrm{~g} / \mathrm{kg} /$ jour. La prise de poids est corrélée à beaucoup de facteurs comme l'existence de complications médicales à l'admission et l'appétit. La mortalité globale de notre échantillon était de 8,1\%. Ce résultat est supérieur à celui de Mbusa Kambal R et al(2016) qui est de 7,5\%. Par contre Mwene- 
Batu P et al (2020) retrouve une mortalité largement supérieur à 15.1\%. Dans une étude rapportée par Singh et al (2014) la mortalité hospitalière était de 1,2\%. Bien que notre taux de mortalité soit élevé, il est inférieur aux 10\% recommandé par le Global SPHERE Standards (2003). Il est largement en dessous de certains rapportés dans une méta-analyse rapportée par Lenters LM et al (2013). Les facteurs de risque de décès étaient le jeune âge, la survenue de complication et l'émaciation sévère. Les études de Munthali et al(2015), de Singh et al (2014) et de Chisti et al (2014) avaient rapporté le jeune âge et l'émaciation comme facteurs de risque importants. La déshydratation sévère, la détresse respiratoire et l'hypothermie sont les principales complications chez nos patients en hospitalisation. Dans notre étude le taux d'abandon était de 1,7\%. Des résultats similaires sont retrouvés par Mbusa Kambale $\mathrm{R}$ et al (2016). Par contre dans la série de Mwene-Batu P et al (2020) le taux d'abandon est plus élevé à 32,6\%. L'abandon du traitement est souvent motivé par la pauvreté de la famille, le manque d'amélioration du patient. Dans notre unité les enfants sortis sous avis médicale ont droit à un sac de riz, de maïs et une certaine quantité d'huile. Cela peut encourager les parents à ne pas abandonner la récupération nutritionnelle. Les principales limites de notre étude sont : les pesées quotidiennes des enfants sont effectuées par des équipes différentes souvent avec de balances différentes. Cela peut influencer la qualité des pesées.

\section{Conclusion}

Notre étude a montré une prévalence élevée de la malnutrition chez les enfants âgés de moins de 24 mois. Le marasme est la forme prédominante de la malnutrition. Le mois de juillet constitue la période critique d'admission dans notre unité. La mortalité est très importante chez les nourrissons, les patients atteints de marasme et ceux qui ont de complication médicale. Pour bien améliorer la situation il faut promouvoir et encourager l'allaitement maternel exclusif jusqu’à 6 mois, améliorer la pratique de la diversification alimentaire du nourrisson et bien renforcer la prise en charge des complications médicales chez les malnutris aigus sévères.

\section{Conflits d'intérêts}

Les auteurs ne déclarent aucun conflit d'intérêt

\section{Contribution des auteurs}

Djibrilla BONKANO BAOUA, Moumouni KAMAYE, Moumouni GARBA: conception et élaboration du protocole, collecte des données, analyse des données et rédaction de l'article. Moumouni GARBA, Nafissatou NANEITO MOUSSA, Mahaman Sani MAMAN MOUSSA : conception et élaboration du protocole et rédaction de l'article. Djibrilla BONKANO 
BAOUA, Moumouni KAMAYE, Moumouni GARBA : conception et élaboration du protocole et collecte des données.

Tous les auteurs ont lu et approuvé la version finale du manuscrit.

\section{Remerciements}

Les auteurs remercient la direction générale du centre hospitalier régional de Niamey et son unité de statistique qui ont autorisé l'accès aux données du service de CRENI et leur analyse pour la réalisation de l'étude.

\section{Tableaux et figures}

Tableau 1 : caractéristiques sociodémographiques des patients

Tableau 2 : Les valeurs du Z score des patients à l'admission et à la sortie

Tableau 3 : complications au cours de l'hospitalisation

Tableau 4 : causes probables de décès

Figure 1 : répartition mensuelle des admissions

Tableau 1 : caractéristiques sociodémographiques des patients

\begin{tabular}{c|cc}
\multicolumn{1}{c|}{ Nombre } & pourcentage \\
\hline 0-5 mois & 85 & $10,4 \%$ \\
6-11 mois & 264 & $32,4 \%$ \\
12-23 mois & 324 & $39,8 \%$ \\
24 mois et plus & 141 & $17,4 \%$ \\
Masculin & Genre & \\
Féminin & 427 & $52,5 \%$ \\
Zone urbaine & 387 & $47,5 \%$ \\
Zone rurale & Provenance \\
faible & 604 & $74,2 \%$ \\
Moyen & 210 & $25,8 \%$ \\
Elevé & Niveau de vie & $75 \%$ \\
& 611 & $25 \%$ \\
\hline
\end{tabular}

Tableau 2 : Les valeurs du Z score des patients à l'admission et à la sortie Rapport Poids/Taille Nombre Pourcentage

\begin{tabular}{lcl|}
\hline \multicolumn{3}{c}{ A l'admission } \\
$\leq-1,5$ & 8 & $0,98 \%$ \\
$\leq-2$ & 7 & $0,85 \%$ \\
$\leq-3$ & 745 & $91,52 \%$ \\
Normal & 7 & $0,85 \%$ \\
$\geq-2$ & 11 & $1,35 \%$ \\
$\geq-3$ & 36 & $4,42 \%$ \\
& A la sortie \\
$\leq-1,5$ & $\mid$ 25 &
\end{tabular}




$\leq-2$
$\leq-3$
Normal
$\geq-1,5$
$\geq-2$
$\geq-3$

$\begin{array}{|ll|}30 & 3,68 \% \\ 362 & 44,47 \% \\ 23 & 2,82 \% \\ 44 & 5,40 \% \\ 63 & 7,74 \% \\ 267 & 32,80 \%\end{array}$

Tableau 3 : complications au cours de l'hospitalisation

\begin{tabular}{l|ll} 
Complications & Nombre $(N)$ & $\%$ \\
\hline Déshydratation & 216 & 26,53 \\
Détresse respiratoire & 50 & 6,14 \\
Hypothermie & 26 & 3,19 \\
Hypoglycémie & 6 & 0,74 \\
Etat de choc & 5 & 0,61 \\
Aucune complication & 511 & 61,77
\end{tabular}

Tableau 4 : causes probables de décès

\begin{tabular}{l|ll} 
Causes de décès & Nombre & pourcentage \\
\hline Anémie sévère & 4 & $6,06 \%$ \\
Choc cardiogénique & 4 & $6,06 \%$ \\
Choc septique & 15 & $22,72 \%$ \\
Etat de mal convulsif & 4 & $6,06 \%$ \\
Déshydratation sévère & 23 & $34,84 \%$ \\
Hypoglycémie & 3 & $4,54 \%$ \\
Détresse respiratoire & 13 & $19,69 \%$
\end{tabular}

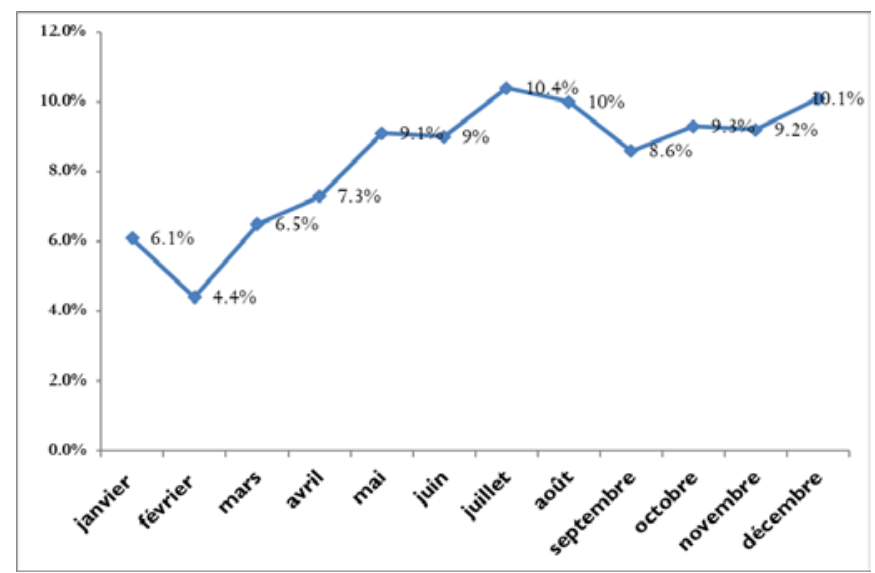

Figure 1 : répartition mensuelle des admissions

\section{References:}

1. UNICEF, WHO (World Health Organization)/World Bank. (2012). Levels and Trends in Child Malnutrition. New York, 
Geneva, and Washington, DC: UNICEF-WHO-The World Bank Joint Child Malnutrition Estimates.

2. Burza S1, Mahajan R1, Marino E1, Sunyoto T1, Shandilya C1, Tabrez M1, Kumari K1, Mathew P1, Jha A1, Salse N1, Mishra KN1 (2015). Community-based management of severe acute malnutrition in India: new evidence from Bihar. The American Journal of Clinical Nutrition, 101(4):847-59. doi: 10.3945/ajcn.114.093294. Epub 2015 Feb 25.

3. United Nations Children's Fund, World Health Organization. (2009). WHO child growth standards and the identification of severe acute malnutrition in infants and children: A joint statement. Geneva: WHO.

4. United Nations Children's Fund, World Health Organization, World Bank Group. (2015). Levels and trends in child malnutrition. Joint child malnutrition estimates. Key findings of the 2015 edition. Geneva: WHO

5. Institut National de la Statistique (INS) et ICF International. (2013). Enquête Démographique et de Santé et à Indicateurs Multiples du Niger 2012. Calverton, Maryland, USA: INS et ICF International.

6. Mwene-Batu, P., Bisimwa, G., Ngaboyeka, G., Dramaix, M., Macq, J., Lemogoum, D., \& Donnen, P. (2020). Follow-up of a historic cohort of children treated for severe acute malnutrition between 1988 and 2007 in Eastern Democratic Republic of Congo. PLOS ONE, 15(3), e0229675. doi:10.1371/journal.pone.0229675

7. Dembélé 1, Sogodogo S2, Kayembé K, Sangho O, Coulibaly CA, Telly N4, Koné Y, Diakité S, Traoré B(2019). Facteurs déterminants la malnutrition aiguë sévère avec complication chez les enfants âgés de moins de 5 ans dans le district sanitaire de Banamba, Mali, 2014-2018. MALI SANTE PUBLIQUE, Décembre 2019. TOME IX Nº02

8. Madzorera I, Duggan C, Berthé F, Grais RF, Isanaka S(2018). The role of dietary diversity in the response to treatment of uncomplicated severe acute malnutrition among children in Niger: a prospective study. BMC Nutrition (2018) 4:35. https://doi.org/10.1186/s40795-018-0242-y

9. Mengesha MM, Deyessa N, Tegegne BS, Dessie Y (2016). Treatment outcome and factors affecting time torecovery in children with severe acute malnutrition treated at outpatient therapeutic care program. Glob Health Action 2016, 9: 30704 http://dx.doi.org/10.3402/gha.v9.30704. 
10. Zoungrana B, Sawadogo PS, Namwin Siourimè Somda, Tapsoba F, Tankoano A, Savadogo A (2019). Performance et coût de la prise en charge de la malnutrition aiguë sévère avec complications à Kaya, Burkina Faso. Pan African Medical Journal.2019;34:145.doi:10.11604/pamj.2019.34.145.17946

11. Nguefack F, Ehouzou M, Kamgaing N, Chiabi A, Eloundou O, Dongmo E, Koki R, Ndombo PO (2015). Caractéristiques cliniques et évolutives de la malnutrition aiguë sévère chez les enfants infectés par le VIH : étude rétrospective sur 5ans. Journal de Pédiatrie et de Puériculture, 28(5), 223-232. doi:10.1016/j.jpp.2015.07.002

12. Saaka M, Osman SM, Amponsem A, Ziem JB, Abdul-Mumin, Akanbong A, Yirkyio P, Yakubu E, Ervin E.(2015). Treatment Outcome of Severe Acute Malnutrition Cases at the Tamale Teaching Hospital. Journal of Nutrition and Metablism, 2015: 641784. doi. 10.1155/2015/641784.

13. Nimpagaritse M, Korachais C, Nsengiyumva G, MacqJ Meessen B. (2019). Addressing malnutrition among children in routine care: how is the Integrated Management of Childhood Illnesses strategy implemented at health centre level in Burundi? BMC Nutrition, 5(1). doi:10.1186/s40795-019-0282-y

14. Chaand I, Horo M, Nair M, Harshana A, Mahajan R, Kashyap V, Dasgupta R (2019). Malnutrition in Chakradharpur, Jharkhand: an anthropological study of perceptions and care practices from India. BMC Nutrition, 5(1). doi:10.1186/s40795-019-0299-2

15. Mbusa Kambale M, Kasengi JB, Mutendela Kivukuto J, Murhula Cubaka L, Masumbuko Mungo B, Bisimwa Balaluka G (2016). Profil infectieux et mortalité des enfants âgés de 0 à 5 ans admis pour malnutrition aiguë sévère: étude de cohorte rétrospective au Centre Nutritionnel et Thérapeutique de Bukavu, République Démocratique du Congo. Pan African Medical Journal. 2016; 23:139 doi:10.11604/pamj.2016.23.139.8370

16. Kanan, SOH. Swar, MO. (2016). Prevalence and outcome of severe malnutrition in children less than five years old in Omdurman Paediatric Hospital, Sudan. Sudanese Journal of Paediatrics, 16(1):23 - 30.

17. Singh KI, Badgaiyan N, Ranjan A, Dixit HO, Kaushik A, Kushwaha KP, Aguayo VM. (2014). Management of Children with Severe Acute Malnutrition: Experience of Nutrition Rehabilitation Centers in Uttar Pradesh, India. Indian pediatrics, $51: 22-25$. 
18. Kaboré A, Ido R, Ouédraogo SAP, Savadogo H, Bélemviré A, Kaboret S, To-gueyeni L, Ouédraogo S, Yé D (2019). Malnutrition aigüe sévère chez les enfants de moins de 6 mois: Prévalence intrahospitalière. $\mathrm{R}$ Revue Africaine et Malgache pour la Recherche Scientifique / Sciences de la Santé / Vol.1, N² 2, juillet 2019Af.

19. Munthali, T. Jacobs, C. Sitali, L. Dambe, R. Michelo, C. (2015). Mortality and morbidity patterns in under-five children with severe acute malnutrition (SAM) in Zambia: a five-year retrospective review of hospital-based records (2009-2013). Archives of Public Health, 73 (1):23 DOI 10.1186/s13690-015-0072-1.

20. Chisti MJ, Graham SM, DukeT et al. (2014 ). Post-Discharge Mortality in Children with Severe Malnutrition and Pneumonia in Bangladesh. PLoS ONE, 9(9): e107663. doi: 10.1371/journal.pone.0107663 .

21. Lenters LM, Wazny K, Webb P, Ahmed T, Bhutta ZA (2013). Treatment of severe and moderate acute malnutrition in low- and middle-income settings: a systematic review, meta-analysis and Delphi process. BMC Public Health 2013, 13(Suppl 3):S23 http://www.biomedcentral.com/1471-2458/13/S3/S23. 